\title{
Multidrug Resistant Tuberculosis involving the Clavicle, Spine and Ribs
}

\author{
H Krishnan, MS Orth, KL Chan, MD \\ Department of Orthopaedic, Queen Elizabeth Hospital, Kota Kinabalu, Malaysia
}

\begin{abstract}
This report describes an unusual case of multidrug resistant tuberculosis (MDR-TB), involving the right clavicle and multicentric aytpical spine involvement without any neurological deficit. The female patient presented with acute onset of right clavicular pain associated with a one-month history of lower backache with constitutional symptoms. The clavicular lesion and MRI spine findings were highly suggestive of TB. Anti TB drugs (ATD) were started empirically as Sabah, Malaysia the patient's home, is an endemic area for TB. Despite, 2 months of ATD administration, the patient did not respond well clinically and developed left sided chest wall abscesses arising from the left 3rd and 6th ribs. She was then treated for MDR-TB infection and has responded well to this treatment.
\end{abstract}

Key Words:

MDR-TB, Clavicular pain, Endemic area.

\section{INTRODUCTION}

Tuberculosis (TB) of the clavicle occurs rarely and is seldom suspected before a biopsy is performed, because tumours are much more common than such infections ${ }^{1}$. Of note, however, spinal tuberculosis accounts for $50 \%$ of the cases of musculoskeletal tuberculosis, $15 \%$ of the cases of extrapulmonary tuberculosis and $2 \%$ of all cases of tuberculosis ${ }^{2}$. Although spinal TB has become relatively rare in developed countries, incidence has been increasing in developing counties. A high index of suspicion is required for spinal tuberculosis even in atypical presentations. With this in mind, we report a case of multidrug resistant TB (MDR-TB) of the clavicle and spine in which the patient did not respond to one month of treatment with 1st line anti-TB drugs (ATD) and subsequently developed left sided chest abscesses arising from the left 3rd and 6th ribs.

\section{CASE REPORT}

A 26-year-old lady presented with complaints of severe right-sided clavicle pain and pain in the lower back of about
1 months duration. Antibiotics and other supportive treatment were started empirically; after one month of antibiotic administration, there was no clinical improvement. The patient reported a history of loss of appetite, weight loss, low grade fever on and off, night sweats, intermittent headache, giddiness and difficulty moving her neck. Upon further questioning, we found neither leg symptoms nor involvement of other systems. Her father was treated for TB with anti TB drugs of the knee four years prior.

A physical examination revealed bony tenderness over the right clavicle but no discharging sinus. There was neck stiffness and reduced range of movement of the cervical and lumbar spine, Kernig's sign was negative, there was no cervical lymphadenopathy or deformity of the spine. She had a BCG (Bacillus Calmette-Guérin (TB vaccination with live virus) scar and neurological examination was normal.

A plain radiography of the chest showed normal lung fields but noted impending pathological fracture with a tumour-like mass invading the middle third of right clavicle (Fig. 1). Plain radiography of the spine revealed a compression fracture at L4. Her erythrocyte sedimentation rate (ESR) was $100 \mathrm{~mm} /$ hour and C-reactive protein (CRP) level was $8.71 \mathrm{mg} / \mathrm{dl}$ (both elevated). Serology for HIV (by ELISA assay) was negative. The tuberculin test was negative and sputum acid-fast bacillus (AFB) was negative. A magnetic resonance imaging (MRI) scan of the entire spine revealed multiple lytic lesions at the centre of the C2, C7, T5, T10, T12, L2 and L4 vertebral bodies, partial collapse of the T5 and L4 vertebral bodies and mild spinal compression at the T5 level (Figure 2). Based on the history, clinical examination, laboratory results, and X-ray and MRI images, the working diagnosis was impending pathological fracture of the right clavicle with noncontagious atypical TB of the spine.

The patient was started on empirical 4 drug (isoniazid, $5 \mathrm{mg} / \mathrm{kg}$; rifampicin, $10 \mathrm{mg} / \mathrm{kg}$; ethambutol, $15 \mathrm{mg} / \mathrm{kg}$; and pyrazinamide, $20 \mathrm{mg} / \mathrm{kg}$ ) ATD chemotherapy one day prior to surgery. The operative procedure consisted of open excision biopsy of the middle third of the right clavicle, bone grafting and locking plate insertion for clavicle stabilization and was 


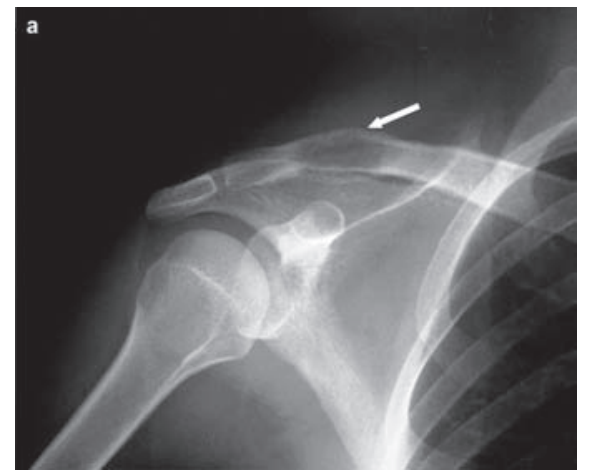

Fig. 1a: Pathological fracture with tumourlike mass evading middle third of right clavicle.

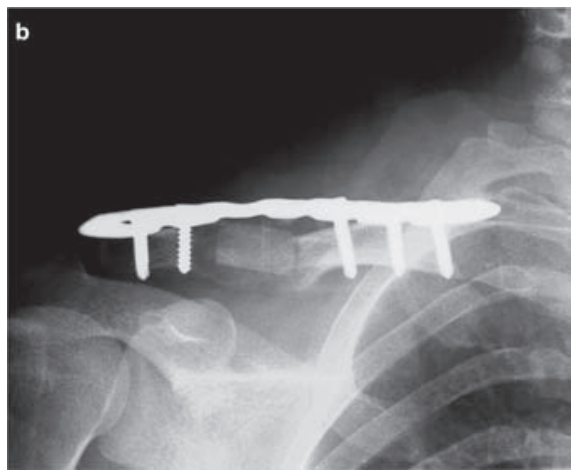

Fig. 1b: Open biopsy of the right middle third of clavicle, bone grafting and locking plate insertion for clavicle stabilization.

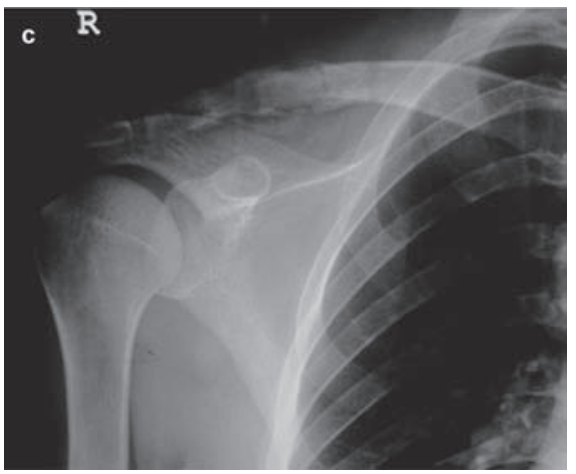

Fig. 1c: The plate and screws were removed after 3 months of therapy because of implant failure.

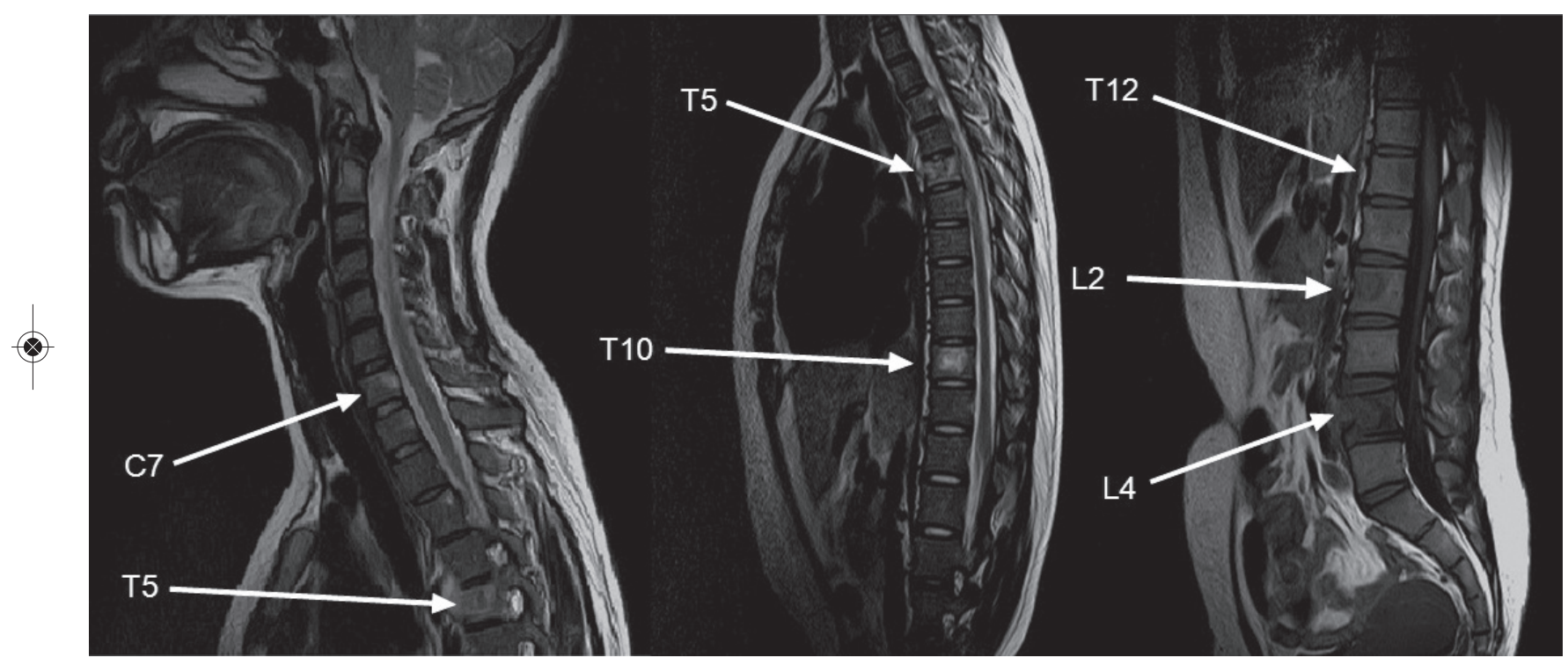

Fig. 2: MRI scan of whole spine revealed multiple lytic lesions are seen at vertebral bodies of C2, C7, T5, T10, T12, L2 and L4; partial collapse of T5 and L4 vertebral bodies with mild spinal compression at T5 level (atypical presentation of TB spine).

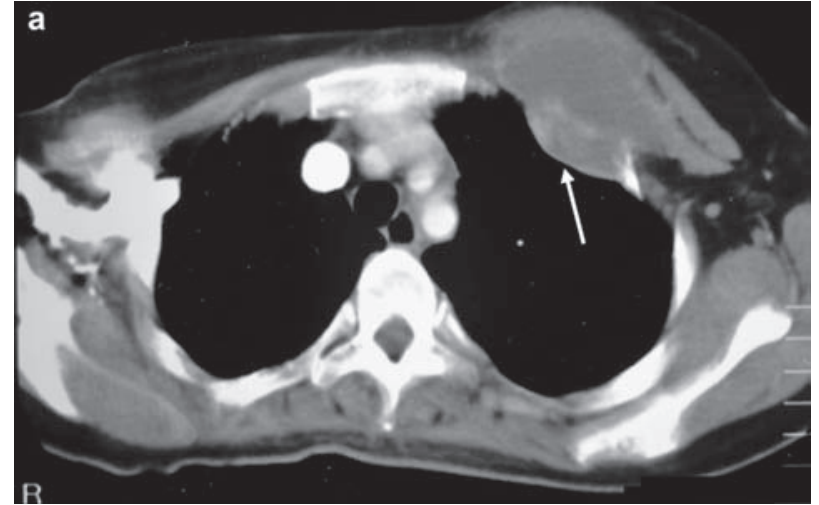

Fig. 3a: Contrast Enhanced CT (CECT) Thorax scan showing (a) abscess collection in the left anterior chest wall that arises from the 3rd rib, encapsulated and does not involve the muscle or pleural.

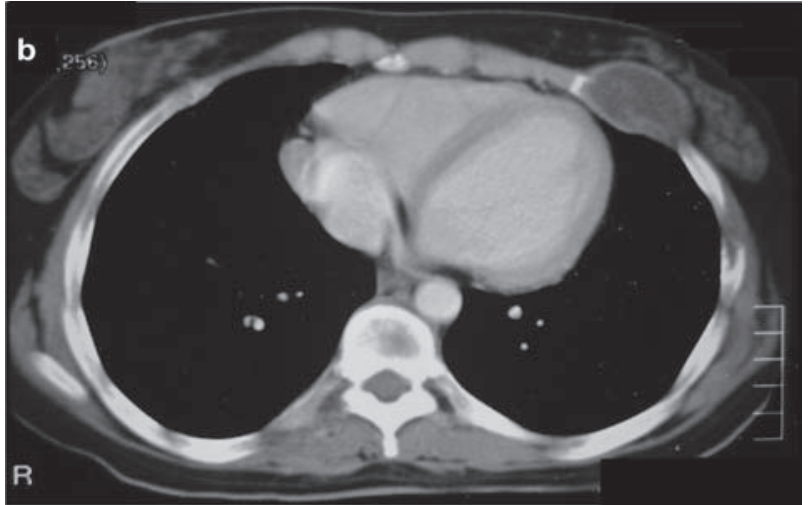

Fig. 3b: Another smaller abscess (b) collection at the level of 6 th rib, just inferior to the breast and seen displacing the breast parenchymal. 
performed at a private medical centre. Clavicular tissue histopathology examination (HPE) showed chronic granulomatous inflammation, Ziehl-Neelsen (ZN) stain for Acid Fast Bacillus (AFB) was negative and no other malignant cells were found.

Despite ATD treatment for a period of 2 months, the patient developed left sided chest and breast swelling, but with no shortness of breath or pus discharge from the left nipple or surrounding skin. Contrast Enhanced CT (CECT) of the thorax (Fig. 3) showed an abscess (size 10x8x9cm) in the left anterior chest wall arising from the $3 \mathrm{rd}$ rib; the abscess was encapsulated and did not involve the muscle or pleura; another smaller abscess was present (size $4 \times 1 \times 2 \mathrm{~cm}$ ) at the level of the 6th rib, just inferior to the breast. Both lung fields were clear and there was no significant mediastinal or other lymphadenopathy and the visualized part of the liver was clear of lesions. The brain CECT showed destruction of the right lateral mass at the $\mathrm{C} 1$ vertebrae but the brain was not involved.

The patient then underwent incision and drainage of the left chest wall cold abscess; all cultures and stains was negative. MDR-TB drug therapy was initiated using second line TB drugs. The plate and screws at the right clavicle were removed after 3 months due to implant failure but the operative site had united. Currently, the patient is doing well and remains on 2nd line ATD therapy. She has been in treatment for 1.5 years with no drug complications and the clavicular lesion has united with no other new lesions or complaints.

\section{DISCUSSION}

The diagnosis of MDR-TB for this patient was established, as the patient had not received ATDs in the past. Secondarily the patient was put on 2nd line ATDs. Resistance to at least the two major ATDs, isoniazid and rifampicin has been termed MDR-TB. She showed good clinical recovery in terms of weight gain, improving appetite and no recurrence of pain or new lesions.

Treatment of MDR-TB requires prolonged and expensive chemotherapy using second-line drugs of heightened toxicity. Should resistance to the second line drugs also arise then the disease becomes virtually untreatable. Drug resistant-tuberculosis (XDR-TB) has been reported extensively in all regions of the world ${ }^{3}$. XDR-TB is defined as resistance to at least rifampicin, isoniazid, a second line injectable drug (capreomycin, kanamycin or amikacin) and a fluoroquinolone.[4] This particular patient was treated for MDR-TB infection and started on second line TB drugs. Following a 6-months intensive phase of 6 drug ATD; pyrazinamide $(40 \mathrm{mg} / \mathrm{kg})$ and ethambuthol $(30 \mathrm{mg} / \mathrm{kg})$ in combination with oral Ciprofloxacin (250mg BD), oral clarithromycin (250mg BD) and oral ofloxacin (400md BD) and 2 months of intravenous amikacin $(15 \mathrm{mg} / \mathrm{kg})$. Subsequently, the patient was treated with maintenance doses of ATD that included pyrazinamide for 6 months and ciprofloxacin, clarithromycin and ofloxacin for 12 months. The ATD was started empirically prior to the surgery, as this region is an endemic area for $\mathrm{TB}^{5}$. (1/3rd of the nation's cases are reported in the Sabah region) and the clinical picture fit the diagnosis of TB.

This report describes a diagnostic dilemma of a patient present with a clavicular lesion and atypical TB spine; MDRTB which was diagnosed after failure of treatment with ATDs The patient did not improve clinically and developed rib TB. 


\section{REFERENCES}

1. Fang JT, Huang CC, Liu HP. Apparent neoplasm of the clavicle of a dialysis patient, ultimately revealed as tuberculosis. Nephrol Dial Transplant 1996; 11: 1380-2.

2. Naim-ur-Rahman. Atypical forms of spinal tuberculosis. J Bone Joint Surg [Br] 1980; 62(2): 162-5.

3. Centers for Disease Control (CDC). Nosocomial transmission of multidrug-resistant tuberculosis among HIV-infected personsFlorida and New York, 1988-1991. MMWR Morb Mortal Wkly Rep 1991; 40: 585-91.

4. Edlin BR, Tokars JI, Grieco MH, Crawford JT, Williams J, Sordillo EM et al. An outbreak of multidrug-resistant tuberculosis among hospitalized patients with the acquired immunodeficiency syndrome. N Engl J Med 1992; 326(23): 1514-21.

5. Dony JF, Ahmad J, Khen Tiong Y. Epidemiology of Tuberculosis and Leprosy Sabah, Malaysia. Tuberculosis 2004; 84: 1-2. 